\title{
The Economic Value of Improved Productivity from Treatment of Chronic Hepatitis C Virus Infection: A Retrospective Analysis of Earnings, Work Loss, and Health Insurance Data
}

\author{
Mark Sulkowski · Raluca Ionescu-Ittu · Dendy Macaulay · Yuri Sanchez-Gonzalez
}

Received: June 29, 2020 / Accepted: September 2, 2020 / Published online: September 14, 2020

(C) The Author(s) 2020

\begin{abstract}
Introduction: Patients with chronic hepatitis $\mathrm{C}$ virus infection (HCV) may incur significant indirect costs due to health-related work loss. However, the impact of curative HCV therapy on work productivity is not well characterized. We estimated the economic value of improved productivity following HCV treatment.

Methods: Adults diagnosed with HCV infection (Optum Healthcare Solutions data; Q1 1999 to Q1 2017) were stratified into two cohorts: (1) treated cohort, patients who received HCV therapy and (2) untreated cohort, therapy-naïve patients. For the treated cohort, the index date
\end{abstract}

Digital Features To view digital features for this article go to https://doi.org/10.6084/m9.figshare.12902408.

Electronic supplementary material The online version of this article (https://doi.org/10.1007/s12325020-01492-x) contains supplementary material, which is available to authorized users.

M. Sulkowski

Department of Medicine, Johns Hopkins University

School of Medicine, Baltimore, MD, USA

R. Ionescu-Ittu $(\bowtie)$

Analysis Group, Inc., Montréal, QC, Canada

e-mail: Raluca.Ionescu-Ittu@analysisgroup.com

D. Macaulay

Analysis Group, Inc., New York, NY, USA

Y. Sanchez-Gonzalez

AbbVie, Inc., Mettawa, IL, USA was set at the end of the post-treatment monitoring period, assumed to be 6 months after the end of treatment for patients with cirrhosis or for those treated with interferon-based therapy, and 3 months after the end of treatment for patients without cirrhosis who received interferon-free therapy. For the untreated cohort, an index date was randomly selected post-HCV diagnosis. Time from the index date to the first work-loss event was assessed using time to event analyses. An economic modeling approach was used to monetize the improved productivity from reduced risk of work-loss event in the 4 years post-index.

Results: Patients in the treated cohort had a lower risk of experiencing a work-loss event compared to untreated patients [unadjusted and adjusted hazard ratios and 95\% CI 0.72 (0.61-0.86), and 0.68 (0.55-0.85), respectively; $p<0.001$ for both]. The mean cumulative added productivity value associated with $\mathrm{HCV}$ treatment was US\$4511 (CI \$2778-\$6278) at 1 year post-index and $\$ 21,429$ $\$ 12,733-\$ 30,199)$ at 4 years post-index.

Conclusion: HCV treatment reduces the risk of work loss resulting in productivity gains for employers and employees. The monetary value associated with these productivity gains is substantial, and, after about 4 years, it is comparable to the wholesale acquisition cost of some direct-acting antiviral regimens in the United States. Employers may derive economic benefits from adopting HCV elimination strategies. 
Keywords: Economic value; Hepatitis C virus treatment; Infectious diseases; Work productivity

\section{Key Summary Points}

Why carry out this study?

Although the clinical benefits of HCV therapies are well documented, there is a paucity of information available regarding the impact of HCV therapies on work productivity.

\section{What was learned from the study?}

Adults newly diagnosed with HCV infection who received HCV therapy during the 1999-2017 study period (primarily with interferon) had significantly lower cumulative risk of having a work-loss event compared to those not treated.

The resulting productivity gains among patients receiving HCV therapy were associated with a monetary value that, after 4 years, was comparable to the wholesale acquisition cost of some directacting antiviral regimens in the United States.

The findings from this study suggest that employers may derive economic benefits from the implementation of $\mathrm{HCV}$ elimination strategies.

\section{DIGITAL FEATURES}

This article is published with digital features to facilitate understanding of the article. To view digital features for this article go to https://doi. org/10.6084/m9.figshare.12902408.

\section{INTRODUCTION}

Chronic hepatitis $\mathrm{C}$ virus infection (HCV) is the leading cause of liver-related death in the United States (US) [1]. With an estimated prevalence of $1.0 \%, \mathrm{HCV}$ infection affects approximately 2.4 million people in the US [2, 3]. From approximately 1992 to 2013, HCV infection was treated exclusively with interferon-based therapy, which required 24-48 weeks of therapy and was associated with an increased risk of adverse events and relatively low rates of sustained virologic response (SVR) or HCV cure [4]. Since 2013, several oral directacting antiviral (DAA) therapies have been approved for use without interferon, some of which provide SVR rates higher than 95\% and with treatment durations as short as 8 weeks [9]. Despite this progress, many persons with $\mathrm{HCV}$ infection remain untreated, contributing to the increasing societal consequences of HCV disease $[10,11]$.

The direct burden of HCV disease is high and encompasses both lower quality of life [12] and high direct medical costs and comorbidities [1], including both hepatic manifestations, such as cirrhosis and liver cancer, and extra-hepatic manifestations, such as kidney disease, diabetes, and heart disease [13-15]. Current literature suggests that persons with $\mathrm{HCV}$ also incur a significant indirect cost burden compared to those without $\mathrm{HCV}$, including incremental health-related work absences [1]. Two previous studies estimated the value of work productivity losses in patients with HCV who did not receive treatment by extrapolating work productivity estimated from questionnaires such as the Work Productivity and Activity Impairment [16-19]. One of these studies estimated that treating all HCV patients with a regimen having very high viral eradication rates would translate to annual productivity gains of $\$ 2.7$ billion over a 1-year time horizon compared to no treatment [18]. The second study estimated that the per patient/year productivity losses were $\$ 4847$ higher in untreated HCV patients relative to matched persons without HCV $(\$ 10,316$ vs. $\$ 5469$ for total indirect cost of absenteeism for productivity loss due to absence from work due 
to medical resource utilization and presenteeism, defined as impaired work productivity while working combined) [19]. However, the impact of HCV treatment on work productivity has not yet been assessed in treated versus untreated HCV patients using real-world work loss and employee earnings data. The objective of this study is to estimate the economic value of HCV treatment for employers and employees, based on differences in the risk of experiencing a long-term work-loss event (i.e., leave of absence, disability leave, early retirement) between persons with HCV infection according to treatment status.

\section{METHODS}

\section{Data Source}

Data were procured from the Optum Healthcare Solutions database and covered the Q1 1999 to Q1 2017 study period. This database contains administrative healthcare claims of over 19 million privately insured individuals covered by 84 self-insured Fortune 500 companies from a range of industries, job classifications, and locations across the US. The data include complete medical and pharmaceutical claims for covered employees and their dependents. Work-loss data including leave of absence, longand short-term disability, and early retirement, as well as earnings are available for the patients employed by 44 companies in the database that report work-loss data in addition to healthcare claims.

\section{Compliance with Ethics Guidelines}

Individual patients are de-identified in the data, and the database is Health Insurance Portability and Accountability Act compliant, and, therefore, the study and use of the data did not require approval and oversight by an institutional review board.

\section{Study Design}

The study investigated two mutually exclusive cohorts of patients diagnosed with HCV: a treated cohort who received HCV therapy with interferon and/or DAAs during the study period and following HCV diagnosis, and an untreated cohort who did not receive HCV therapy during the study period and following HCV diagnosis.

For the treated cohort, the index date was set after both the HCV treatment and the post-HCV treatment monitoring were assumed to be completed, [20] to ensure that the healthcare resource utilization related to treatment and post-treatment monitoring would not confound the comparison between treated and untreated patients. The duration of the posttreatment monitoring period was assumed to be longer for patients with a history of cirrhosis or those who received interferon-based therapy. Specifically, the index date was defined as 6 months after the end of treatment for patients with a history of cirrhosis or those who received interferon-based therapy, and as 3 months after the end of treatment for patients without a history of cirrhosis who were treated with interferon-free therapy (i.e., DAAs approved in 2013 or afterwards). For the untreated cohort, the index date was randomly selected among all dates at least 147 days after the patient's first observed HCV diagnosis, which corresponded to the minimal difference between first $\mathrm{HCV}$ diagnosis and index date in the treated cohort.

The 6-month period prior to the index date, inclusive of the index date, was defined as the baseline period. The time period spanning from the index date until the earliest of loss of continuous insurance eligibility, reaching age 65 years, or end of data availability on March 31, 2017 was defined as the follow-up period. Patient characteristics were measured during the baseline period. Study outcomes including work loss and economic value of improved productivity associated with $\mathrm{HCV}$ treatment were measured during the follow-up period and do not include the medically-related absenteeism for treatment and post-treatment monitoring. The follow-up period was censored 4 years post-index as few patients were followed beyond this date. 


\section{Study Sample}

The study sample included adults between 18 and 65 years of age diagnosed with HCV, a washout of $\geq 6$ months with continuous enrollment, and no HCV treatment prior to the first HCV diagnosis was applied. All patients were primary policy holders and actively employed for $\geq 6$ months before and after the index date by the 44 companies which reported work loss data, including long- and short-term disability claims. A sample selection flowchart with specific inclusion/exclusion criteria is presented in Figure S1.

\section{Measures}

Baseline characteristics measured during the baseline period included age, sex, region of residence, index year, Charlson Comorbidity Index (CCI), health plan type, liver cirrhosis, mean days of medically-related absenteeism, and annual salary at the index date. Days of medically-related absenteeism during the baseline period were assessed by imputing a full day of work loss for each inpatient or emergency room visit during the baseline period, and by imputing a half-day of work loss for each outpatient visit during the baseline period, as observed in the claims data. Salary in the month of the index date was annualized and inflated to 2017 USD using the medical component of the Consumer Price Index for inclusion in the economic evaluation. For the treated cohort, the class of index HCV therapy (i.e., DAA vs. interferon treatment) was described.

For the analysis of time to first work-loss event, work loss was captured as reported on a monthly basis by the employer and defined as any of the following: leave of absence, shortterm disability, long-term disability, and early retirement. Definitions of short- and long-term disability vary across employers, with shortterm disability usually ranging between 9 and 52 weeks, and long-term disability reflecting any disability leave that extends beyond the employer's short-term disability limit. Time from the index date to the first work-loss event was measured in days and work loss was assumed to start on the first day of the first month post-index when the employer reported a work-loss event.

\section{Statistical Analyses}

Descriptive statistics were calculated for baseline characteristics including means, standard deviations, frequencies, and proportions. Statistical comparisons between cohorts at baseline were conducted using Wilcoxon rank-sum tests for continuous variables and Chi-square tests for categorical variables.

To account for varying follow-up time across patients, the time from index date to the first work-loss event was compared between the treated and untreated cohorts using time to event analyses, including Kaplan-Meier (KM) plots and Cox proportional hazards regression models. From the former, we report the cumulative incidence of work loss (1-KM) in the treated and untreated cohorts with the corresponding value of the log-rank test. From the latter, we report unadjusted and adjusted hazard ratios for the treated versus untreated cohorts, with adjusted hazard ratios controlling for age, sex, region of residence, index year, CCI, cirrhosis, and days and costs of medicallyrelated absenteeism in the baseline period.

In addition, the work-loss cumulative incidence (1-KM) plot was also used to extract one of the inputs needed for economic valuation of work productivity. Specifically, for each day after the index date, the gap between the cumulative incidence curves in treated and untreated cohorts (i.e., the difference in risk of work-loss event between the cohorts) was used to quantify the potential reduction in work loss attributable to HCV treatment.

The value of work productivity, or value of avoiding work loss, at each day during the follow-up was assessed using an economic valuation approach [21] based on the cross-product between (1) the difference in risk of work-loss event between the cohorts at each day during the follow-up and (2) the average daily salary of the treated cohort, inferred by dividing the average annualized salary of the treated cohort at the index date by the number of days in a 
year. This cross-product between salary and work-loss risk at each day during the follow-up corresponded to an average dollar estimate of the incremental daily productivity among treated patients due to not experiencing a work-loss event and remaining in the workforce. The economic evaluation relied on the patients' salary at the index date because salary during the follow-up could have been subject to variations due to exogenous factors and confound the estimates.

To provide an average cumulative monetary estimate of productivity gains associated with HCV treatment over time, the average dollar estimate of the incremental daily productivity of treated patients was then accumulated and reported at 1 year and 4 years post-index date. Bootstrap resampling methods were utilized to generate confidence intervals for the average incremental daily productivity estimates for the first year post-index and the full 4 years followup post-index.

\section{RESULTS}

A total of 3052 eligible $\mathrm{HCV}$ patients were identified: $673(22.0 \%)$ were treated for $\mathrm{HCV}$ and $2379(77.9 \%)$ were untreated. Unadjusted baseline demographic and clinical characteristics are presented in Table S1. Baseline salary was similar for treated versus untreated patients [mean (SD): $\quad \$ 72,008.70 \quad(\$ 50,708.38) \quad$ vs. $\$ 69,160.23$ (\$53,319.87); $p=0.2668$ ]. Patients in the treated cohort had higher rates of cirrhosis than patients in the untreated cohort at baseline (treated: 8.3\%; untreated: 6.1\%; $p=0.0442$ ) and more days of medically-related absenteeism in the baseline period, which included a post-treatment monitoring period that was treatment-specific [mean (SD) number of days of medically-related absenteeism in treated: 4.2 (4.5); untreated: 3.0 (4.3); $p<0.0001]$. The treated cohort also included fewer females (treated: 33.3\%; untreated: $38.5 \%$; $p=0.0127)$. Significant differences were also observed between the treated and untreated cohorts with respect to index year and region. Among patients in the treated cohort, $32(4.8 \%)$ were on a DAA treatment.
Treated patients had a lower cumulative risk of having a work-loss event than untreated patients at all points during the 4-year followup period [HR (95\% CI) $0.72 \quad(0.61-0.86)$, $p=0.0002$; Fig. 1]. After adjusting for confounding factors, treated patients had a lower hazard of work loss [HR $\left(\begin{array}{llll}95 \% & \text { CI }) & 0.68\end{array}\right.$ (0.55-0.85), $p=0.0006$; Fig. 1]. In the multivariate Cox regression model, other factors associated with increased hazard of work loss included age, female sex higher overall burden of disease (CCI), presence of cirrhosis, and more days of medically-related absenteeism in the baseline period (Table S2).

The potential reduction in work loss attributable to HCV treatment (i.e., gap between treated and untreated patients in the work-loss KM curves) varied over time in the $3-10 \%$ range (Fig. S2). The mean work productivity value associated with HCV treatment was $\$ 4511$ (CI \$2778-\$6278) at 1 year post-index and $\$ 21,429$ (CI $\$ 12,733-\$ 30,199$ ) at 4 years post-index (Fig. 2). This corresponded to an average annual productivity benefit from $\mathrm{HCV}$ treatment of $\$ 5357$ per patient/year over the 4-year period.

\section{DISCUSSION}

The impact of HCV treatment on work productivity has been incompletely characterized. The present study addressed this knowledge gap by estimating the economic value of $\mathrm{HCV}$ treatment for employers and employees based on differences in the risk of work loss between treated and untreated patients with $\mathrm{HCV}$ infection. In the present study, patients with $\mathrm{HCV}$ who underwent treatment had a lower risk of work loss compared to untreated patients (adjusted HR 0.68) over a period of up to 4 years of follow-up. This lower risk of work loss was associated with a productivity value of $\$ 4511$ at 1 year post-index and $\$ 21,429$ at 4 years post index.

Our findings suggest the potential for an economic benefit of HCV treatment for both employers and employees due to avoidance of work-loss events and corresponding work productivity gain among employed patients with 


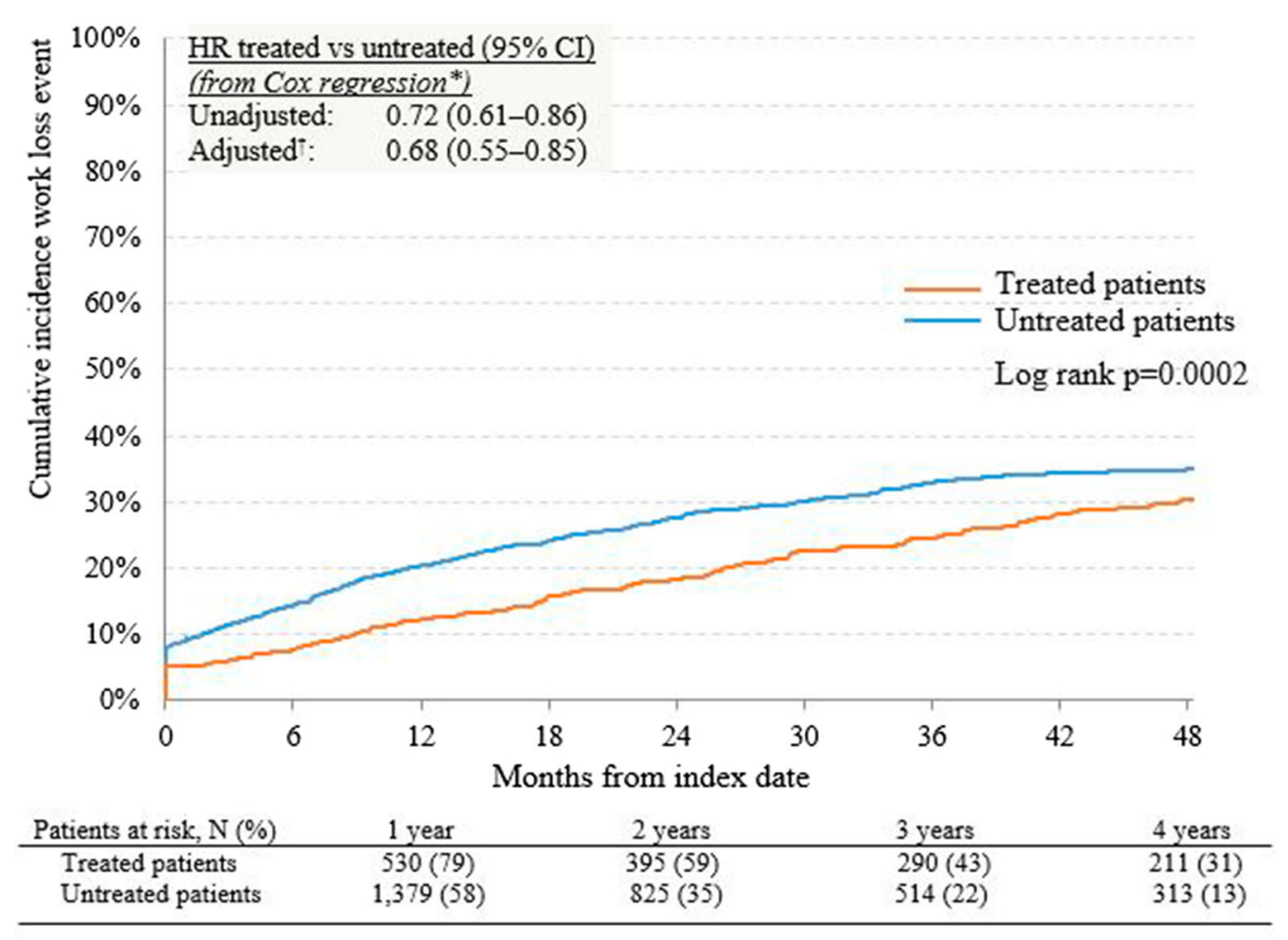

Fig. 1 Time to first work loss in treated versus untreated patients with HCV. *Hazard ratios were based on regression analyses detailed in Supplementary Table 2. $\dagger$ Adjusted for age, sex, region of residence, index year,

HCV who received versus did not receive treatment; this observation is consistent with the findings of other studies. The estimated productivity gain associated with $\mathrm{HCV}$ treatment in the present study $(\$ 4511)$ is of a similar magnitude to the productivity losses reported in a 2012 patient survey for patients with untreated HCV (\$4847) [19]. Taken together, these findings suggest that treatment may correct lost productivity in persons with $\mathrm{HCV}$ infection rendering them similar to levels of productivity observed in persons without $\mathrm{HCV}$ infection. A caveat is that the latter findings may have been subject to selection bias as participants' HCV status was determined through survey methodology [19] rather than recorded diagnoses as in the present study. The survey [19] may also have been subject to confounding effects due to uncontrolled differences between persons with untreated $\mathrm{HCV}$ infection and
CCI, cirrhosis, and days and costs of medically-related absenteeism in the baseline period (Supplementary Table 2). $C I$ confidence interval, $H C V$ chronic hepatitis $\mathrm{C}$ virus infection, $H R$ hazard ratio

uninfected controls from the general population.

In another US analysis, the achievement of SVR with treatment was projected to translate into an annual savings of $\$ 2.7$ billion for the US economy as a whole [18], or an average annual savings of $\$ 1874$ per patient/year [22]. In a study of five European countries, the achievement of SVR with treatment was associated with an average annual gain of 900 euros per employed patient with HCV infection [22]. The estimated average annual savings per patient/ year in both of these prior studies are lower than the estimates from the current study ( $\$ 5357$ per patient/year over 4 years). However, the results of the current study are not directly comparable to these previous estimates for several reasons. First, the previous studies considered lost productivity costs due to both absenteeism, specifically absence from work due to medical resource utilization, and presenteeism defined 


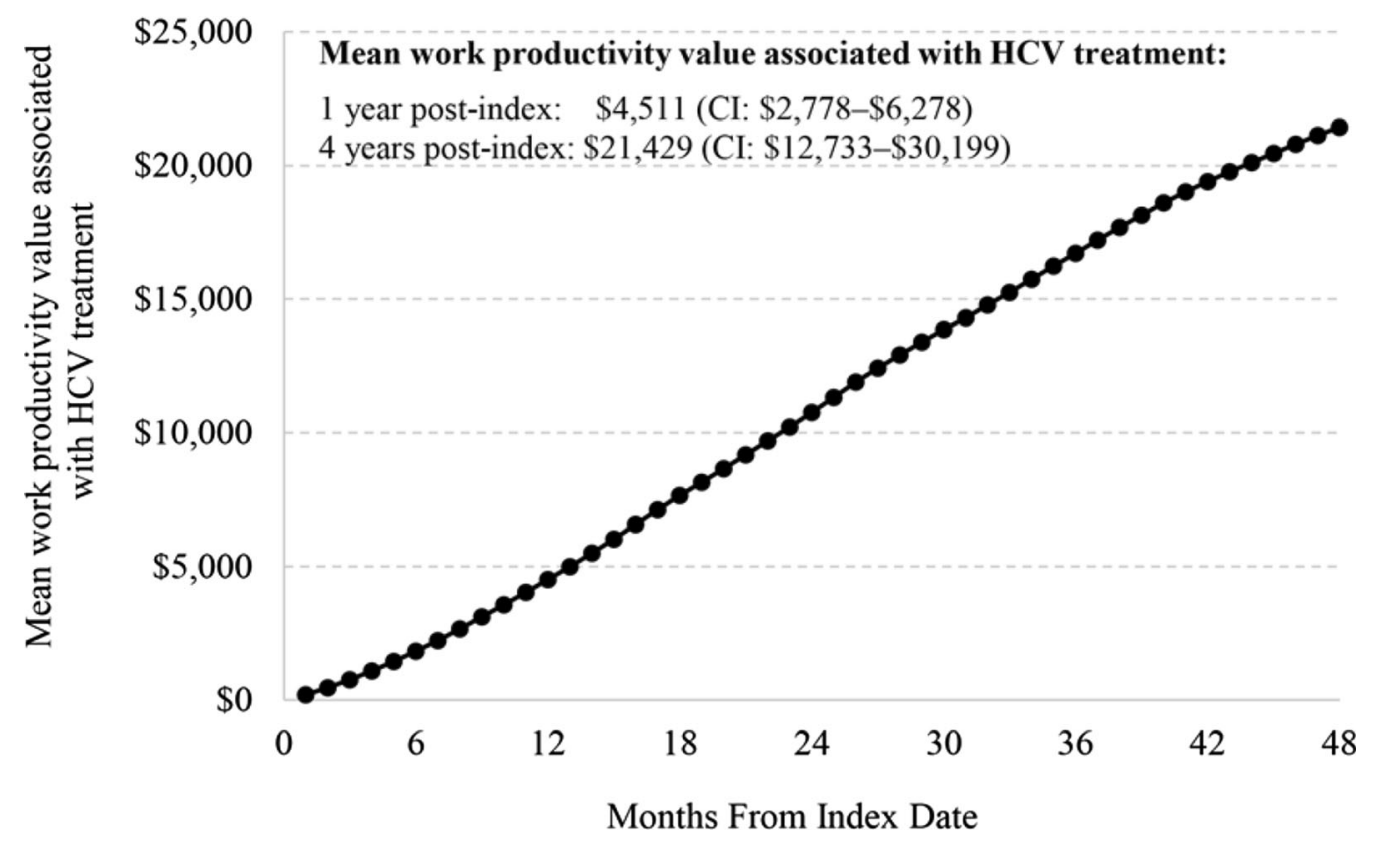

Fig. 2 Mean employer work productivity value associated with HCV treatment; the value of avoiding a work-loss event was inferred by applying the average salary at the index date (converted into a daily salary) for the treated

as reduced productivity while at work [18, 22], while the current study focused on patients' first long-term work-loss event including leave of absence, short- or long-term disability, and early retirement. Second, both previous studies estimated work productivity using the Activity Index-Specific Health Problem instrument $[18,22]$, while the current study assessed work loss directly from the employer records, reflecting the employer perspective with respect to productivity. Finally, work productivity was estimated among participants in HCV clinical trials $[18,22]$, while the current study included employees from select self-insured Fortune 500 companies in the US.

In addition to their economic implications, the present study findings are relevant to the clinical and humanistic burden of HCV disease. Impaired work productivity is a factor associated with poorer health-related quality of life among untreated HCV patients [19]. Patients who respond to HCV treatment have higher productivity and better quality of life compared to those who do not achieve SVR [12]. Thus, the added economic value of HCV treatment might cohort to the cumulative work-loss risk over the follow-up period. $C I$ confidence interval, $H C V$ chronic hepatitis $\mathrm{C}$ virus

reinforce improved physical and emotional well-being among patients.

It is important to note that the present study may have underestimated the total productivity value of $\mathrm{HCV}$ treatment due to its observation period, which spanned from 1999 to 2017. Before 2013, most HCV treatments were interferon-based, whereas more effective and better tolerated oral DAA-based regimens are the standard of care today $[5,6,9]$. Given that only $5 \%$ of the treated patients in the present study received interferon-free treatments, the findings may not be fully generalizable to the present population. However, any beneficial effects observed for interferon-based therapies on work loss are expected to hold or be even greater for better-tolerated oral DAA therapies, which have a superior efficacy and safety profile.

The present study analyzed time to the first work-loss event, without accounting for the total duration of the work-loss event, the risk of subsequent work-loss events, or medically-related absenteeism post-index. To the extent that absence of HCV treatment not only increases the risk of a work-loss event but also lengthens 
the periods of work loss and/or increases susceptibility to absenteeism or future work-loss events, the present results could underestimate the total productivity value of treatment. Moreover, the primary outcome of the present study is work loss and not absenteeism. Important areas for future research may include the impact of newer DAA treatments on the total duration of work-loss events, the risk of subsequent events, absenteeism post-index, and different types of work loss (e.g., presenteeism) in patients with HCV infection.

The present study was subject to certain limitations inherent in observational studies. One cannot rule out the impact of unmeasured confounding variables, such as patient characteristics that are not available in claims databases. These studies are also susceptible to information bias, including biases in reporting. Work-loss data in the present study were reliant on the reporting of work-loss events by various employers, who may have had differing policies governing access to leave of absence, disability, and early retirement. This reporting bias may have introduced unexplained variability into the results, although it is expected to have affected both treated and untreated cohorts equally.

In the present analysis, the $\mathrm{KM}$ rate inputs used to monetize the value of avoiding work loss were not adjusted for baseline differences between the treated and untreated cohorts. Some differences were indeed observed at baseline, including higher rates of cirrhosis, fewer females, and more days of medically-related absenteeism among patients in the treated cohort. However, the unadjusted and adjusted multivariate Cox proportional-hazards regressions yielded similar hazards of work loss (Fig. 1), suggesting that they were unlikely to have confounded the KM rate inputs that were used to monetize the value of avoiding work loss.

There were also some limitations inherent in the economic valuation approach adopted in the present study. When calculating the cost savings associated with treatment, an underlying assumption is that mean annualized salary at the index date remains constant throughout the 4 years follow-up period. In reality, the mean annualized salary varies over time due to both temporal changes in earnings at population level, as well as differences between the patients with short and long follow-up in the data (the former will not contribute to the estimation in the later years of the data). Additionally, only one economic cost equivalent to a patient's salary was applied to all types of workloss events. However, from the employer perspective, the risk of absence from the workforce may have different values depending on the expected duration of the event (e.g., leave of absence vs. disability).

Finally, it is worth considering that productivity loss costs may be perceived and accrued differently by employed persons with $\mathrm{HCV}$ infection relative to employers, or society at large. The productivity value may be shared between the employer and the employed HCV patient, and may vary depending on the type of work-loss event. From the patient perspective, disability payments could compensate for a portion of lost earnings, which may or may not be paid by the employer depending on the type of work loss. However, the employer would still incur the lost productivity. We note that the employed persons with $\mathrm{HCV}$ in the present study were covered by large US-based self-insured employers. Therefore, their average annual salary was likely higher than that of the general workforce. Applying the present economic valuation approach to a lower-income population could potentially reveal a reduced economic burden associated with work loss due to untreated HCV.

From a societal perspective, the achievement of SVR may provide considerable benefits by reducing the negative externalities associated with HCV infection. Consistent with this, one study projected that screening the entire US population and treating all persons with $\mathrm{HCV}$ infection would result in net cost savings for society in the long term, with screening and treatment costs fully offsetting the substantial economic burden associated with untreated $\mathrm{HCV}$ infection [23]. However, further work is still needed to identify cost-effective strategies for optimizing the continuum of care from screening to treatment among employed persons with HCV disease [24]. 


\section{CONCLUSIONS}

In the present study, patients with HCV infection who underwent antiviral treatment had a lower risk of work loss compared to those who were not treated, which translated into an economic benefit to employers and employees from greater work productivity. These findings suggest that some direct costs associated with $\mathrm{HCV}$ treatment may be at least partially offset by work productivity gains. We note that the monetary value associated with HCV-related work loss is substantial, and, after about 4 years, it is comparable to the wholesale acquisition cost of current, oral DAA regimens [25]. Employers should consider this productivity value from $\mathrm{HCV}$ treatment an additional benefit beyond the medical cost savings from reducing hepatic and extrahepatic health risks to support a robust economic rationale for adopting HCV elimination strategies in their workplaces.

\section{ACKNOWLEDGEMENTS}

Funding. Sponsorship for this study and Rapid Service Fee were funded by AbbVie. The study sponsor was involved in all stages of the study research and manuscript preparation. No honoraria or payments were made for authorship.

Medical Writing, Editorial, and Other Assistance. Medical writing assistance in the preparation of this article was provided by Michaela Stamm and Mona Lisa Chanda, employees of Analysis Group, Inc. Support for this assistance was funded by AbbVie. The authors would like to thank Darren Thomason and Olivia Liu from Analysis Group for analytical support. Financial support for these services was provided by AbbVie.

Authorship. All named authors meet the International Committee of Medical Journal Editors (ICMJE) criteria for authorship for this article, take responsibility for the integrity of the work as a whole, and have given their approval for this version to be published.

Authorship Contributions. All authors contributed to the development of the publication and maintained control over the final content.

Disclosures. Dr. Mark Sulkowski is an employee of Johns Hopkins University School of Medicine and reports research grants from AbbVie, Gilead, Janssen, AssemblyBio, Proteus Digital Health and the National Institutes of Health and serves as a consultant for AbbVie, Gilead, Immunocore, Arbutus, Biomarin and AssemblyBio. Dr. Raluca Ionescu-Ittu reports working for Analysis Group, which received consulting fees from AbbVie, during the conduct of the study. Dr. Dendy Macaulay reports working for Analysis Group, which received consulting fees from AbbVie, during the conduct of the study. Dr. Yuri Sanchez Gonzalez is an employee of AbbVie Inc. and may own stocks and/or options of the company.

Compliance with Ethics Guidelines. Individual patients are de-identified in the data and the database is Health Insurance Portability and Accountability Act compliant, and, therefore, the study and use of the data did not require approval and oversight by an institutional review board.

Data Availability. Access to the database used in this study was licensed by Analysis Group, Inc. from Optum Healthcare Solutions (https://www.optum.com/business/contact. html), Inc. and is not publicly available.

Open Access. This article is licensed under a Creative Commons Attribution-NonCommercial 4.0 International License, which permits any non-commercial use, sharing, adaptation, distribution and reproduction in any medium or format, as long as you give appropriate credit to the original author(s) and the source, provide a link to the Creative Commons licence, and indicate if changes were made. The images or other third party material in this article are included in the article's Creative Commons licence, unless indicated otherwise in a credit 
line to the material. If material is not included in the article's Creative Commons licence and your intended use is not permitted by statutory regulation or exceeds the permitted use, you will need to obtain permission directly from the copyright holder. To view a copy of this licence, visit http://creativecommons.org/licenses/by$\mathrm{nc} / 4.0 /$.

\section{REFERENCES}

1. Su J, Brook RA, Kleinman NL, Corey-Lisle P. The impact of hepatitis $\mathrm{C}$ virus infection on work absence, productivity, and healthcare benefit costs. Hepatology. 2010;52(2):436-42.

2. Hofmeister MG, Rosenthal EM, Barker LK, Rosenberg ES, Barranco MA, Hall EW, et al. Estimating prevalence of hepatitis $C$ virus infection in the United States, 2013-2016. Hepatology. 2019;69(3): 1020-31.

3. Centers for Disease Control and Prevention. 2018 Hepatitis C Prevalence Estimates 2013-2016. https://www.cdc.gov/nchhstp/newsroom/2018/ hepatitis-c-prevalence-estimates.html. Accessed 17 Jan 2020

4. Drenth JP. HCV treatment-no more room for interferonologists? N Engl J Med. 2013;368(20): 1931-2.

5. Gilead Sciences I. 2019 Sovaldi: Highlights of prescribing information. https://www.gilead.com/ / media/Files/pdfs/medicines/liver-disease/sovaldi/ sovaldi_pi.pdf. Accessed 5 Feb 2020

6. Gilead Sciences I. 2016 Harvoni: Highlights of prescribing information. https://www.gilead.com/ / media/files/pdfs/medicines/liver-disease/harvoni/ harvoni_pi_old.pdf?la=en. Accessed 5 Feb 2020

7. Solbach $\mathrm{P}$, Wedemeyer $\mathrm{H}$. The new era of interferon-free treatment of chronic hepatitis C. Viszeralmedizin. 2015;31(4):290-6.

8. Nkuize M, Serste T, Buset M, Mulkay JP. Combination ledipasvir-sofosbuvir for the treatment of chronic hepatitis $\mathrm{C}$ virus infection: a review and clinical perspective. Ther Clin Risk Manag. 2016;12: 861-72.

9. Afdhal N, Zeuzem S, Kwo P, Chojkier M, Gitlin N, Puoti M, et al. Ledipasvir and sofosbuvir for untreated HCV genotype 1 infection. N Engl J Med. 2014;370(20):1889-988.
10. Park H, Wang W, Henry L, Nelson DR. Impact of alloral direct-acting antivirals on clinical and economic outcomes in patients with chronic hepatitis C in the United States. Hepatology. 2019;69(3): 1032-45.

11. Njei B, Esserman D, Krishnan S, Ohl M, Tate JP, Hauser RG, et al. Regional and rural-urban differences in the use of direct-acting antiviral agents for hepatitis C Virus. Med Care. 2019;57(4):279-85.

12. John-Baptiste AA, Tomlinson G, Hsu PC, Krajden $M$, Heathcote EJ, Laporte $A$, et al. Sustained responders have better quality of life and productivity compared with treatment failures long after antiviral therapy for hepatitis C. Am J Gastroenterol. 2009;104(10):2439-48.

13. Westbrook RH, Dusheiko G. Natural history of hepatitis C. J Hepatol. 2014;61(1 Suppl):S58-68.

14. Negro F, Forton D, Craxi A, Sulkowski MS, Feld JJ, Manns MP. Extrahepatic morbidity and mortality of chronic hepatitis C. Gastroenterology. 2015;149(6):1345-60.

15. Younossi ZM, Kanwal F, Saab S, Brown KA, El-Serag $\mathrm{HB}$, Kim WR, et al. The impact of hepatitis C burden: an evidence-based approach. Aliment Pharmacol Ther. 2014;39(5):518-31.

16. Reilly MC, Zbrozek AS, Dukes EM. The validity and reproducibility of a work productivity and activity impairment instrument. Pharmacoeconomics. 1993;4(5):353-65.

17. 2019 WPAI General Information. https://www. reillyassociates.net/WPAI_General.html. Accessed 11 Dec 2019

18. Younossi ZM, Jiang Y, Smith NJ, Stepanova M, Beckerman R. Ledipasvir/sofosbuvir regimens for chronic hepatitis $\mathrm{C}$ infection: insights from a work productivity economic model from the United States. Hepatology. 2015;61(5):1471-8.

19. El Khoury AC, Vietri J, Prajapati G. The burden of untreated hepatitis C virus infection: a US patients' perspective. Dig Dis Sci. 2012;57(11):2995-3003.

20. George SL, Bacon BR, Brunt EM, Mihindukulasuriya KL, Hoffmann J, Di Bisceglie AM. Clinical, virologic, histologic, and biochemical outcomes after successful HCV therapy: a 5-year follow-up of 150 patients. Hepatology. 2009;49(3):729-38.

21. Etzioni RD, Feuer EJ, Sullivan SD, Lin D, Hu C, Ramsey SD. On the use of survival analysis techniques to estimate medical care costs. J Health Econ. 1999;18(3):365-80. 
22. Younossi Z, Brown A, Buti M, Fagiuoli S, Mauss S, Rosenberg $\mathrm{W}$, et al. Impact of eradicating hepatitis $\mathrm{C}$ virus on the work productivity of chronic hepatitis $\mathrm{C}(\mathrm{CH}-\mathrm{C})$ patients: an economic model from five European countries. J Viral Hepat. 2016;23(3): 217-26.

23. Younossi Z, Blissett D, Blissett R, Henry L, Younossi $\mathrm{Y}$, Beckerman $\mathrm{R}$, et al. In an era of highly effective treatment, hepatitis C screening of the United States general population should be considered. Liver Int. 2018;38(2):258-65.
24. Younossi Z, Papatheodoridis G, Cacoub P, Negro F, Wedemeyer H, Henry L, et al. The comprehensive outcomes of hepatitis $\mathrm{C}$ virus infection: a multifaceted chronic disease. J Viral Hepat. 2018;25(Suppl 3):6-14.

25. 2019 Glecaprevir/pibrentasvir: RED BOOK Product Details. https://www.micromedexsolutions.com/. Accessed 8 Jan 2019 